\title{
Chapitre 2 \\ Les différents types de réacteurs de recherche, situation globale dans le monde, utilisations et risques associés
}

\subsection{Types de réacteurs de recherche}

\section{Différents types adaptés aux applications visées}

Les réacteurs de recherche peuvent être classés selon leurs finalités ou applications, les différences de conception de ces réacteurs en résultant. En pratique, on peut distinguer :

- les réacteurs d'irradiation ou dits MTR (Material Testing Reactor), qui sont destinés principalement à des études et des tests de divers matériaux et combustibles nucléaires, notamment ceux utilisés pour les réacteurs de puissance, ou encore à la production de radioisotopes à usage médical ;

- les réacteurs équipés de " canaux neutroniques " ${ }^{7}$, souvent appelés « à faisceaux sortis de neutrons », qui sont dédiés essentiellement à la recherche fondamentale, par exemple pour des expériences de diffraction neutronique ;

7. Dans le cadre du présent document, les expressions suivantes seront utilisées : un canal neutronique désignera l'ensemble constitué : pour la partie en piscine du réacteur, d'un « doigt de gant », dont l'extrémité (dans la cuve d'eau lourde et au plus près du cœur) sera appelée « nez » et, pour la partie hors piscine (dans le hall des expérimentateurs), d'un « guide de neutrons». 
- les maquettes (ou assemblages) critiques, de très faible puissance thermique, qui sont utilisées notamment pour la détermination de données nucléaires servant à la validation de logiciels de simulation de neutronique des cœurs (de réacteurs de puissance ou de recherche) ;

- les réacteurs d'études de sûreté, qui sont spécifiquement utilisés pour étudier des situations accidentelles représentatives de celles susceptibles de se produire dans des réacteurs de puissance, telles qu'une perte de refroidissement du combustible ou une insertion de réactivité ${ }^{8}$ qui pourraient entraîner sa fusion et le relâchement de produits de fission dans l'environnement ;

- les réacteurs d'enseignement, qui sont généralement de faible puissance thermique et sont utilisés pour la formation du personnel de l'industrie nucléaire ainsi que pour l'enseignement universitaire.

\section{Diversité des conceptions}

Il existe une large gamme de types de conception pour les réacteurs de recherche, contrairement aux réacteurs de puissance pour lesquels un nombre restreint de types de conception s'est progressivement imposé. Parmi la grande diversité des réacteurs de recherche, on peut y distinguer deux groupes principaux que sont :

- les réacteurs à eau lourde ${ }^{9}$ (utilisée comme fluide réfrigérant, modérateur ou réflecteur neutronique),

- les réacteurs refroidis et modérés à l'eau légère.

Parmi les autres types de réacteurs de recherche, on peut citer ceux qui sont modérés neutroniquement par du graphite, les réacteurs homogènes utilisant une solution de nitrate ou de sulfate d'uranium ainsi que les réacteurs à neutrons rapides qui ne

8. Pour caractériser le risque d'emballement d'un cœur, on utilise la notion de réactivité : c'est une grandeur (désignée par $\rho$ ) permettant de mesurer l'écart d'un cœur par rapport à son état juste critique $(\rho=0)$.Typiquement, le retrait ou l'éjection intempestive d'une barre de contrôle ou de sécurité d'un réacteur nucléaire, hors de la zone du cœur, va, par la réduction de la proportion des matériaux absorbants neutroniques dans le cœur, se traduire par un surcroît de " réactivité » du cœur et donc une augmentation de sa puissance, au moins localement. Si cet événement n'est pas maîtrisé par les systèmes de surveillance et de protection du réacteur, il peut conduire à un endommagement significatif voire à la fusion d'éléments combustibles du cœur. Les expressions " insertion », « injection », " introduction » ou " apport » sont indifféremment utilisées. L'expression " excursion de puissance » désigne le transitoire de puissance provoqué par une insertion de réactivité.

9. Pour les réacteurs utilisant du combustible à base d'uranium 235 , ce sont les neutrons lents de faible énergie (optimum pour $E=0,025 \mathrm{eV}$ ) qui ont la plus forte probabilité de produire des fissions. Avec un modérateur efficace, les neutrons issus de la fission de l'uranium 235 sont ralentis jusqu'à ce que leur énergie cinétique soit à peu près égale à l'énergie d'agitation thermique du milieu diffusant $(0,025 \mathrm{eV}$ à la température de $300 \mathrm{~K})$, sans être absorbés. La plupart des fissions se produisent alors à cette énergie et le réacteur est dit à neutrons thermiques. Des fluides modérateurs sont ainsi utilisés ; l'eau lourde $\left(D_{2} O\right)$ est le meilleur modérateur devant, par ordre décroissant, le graphite, le béryllium et l'eau légère $\left(\mathrm{H}_{2} \mathrm{O}\right)$. 
nécessitent pas de modérateur, mais qui peuvent utiliser comme combustible un mélange d'oxyde d'uranium et d'oxyde de plutonium.

- Les réacteurs à eau lourde :

Les réacteurs à eau lourde sont de type « cuve dans une piscine » (tank in pool reactor type). Ils permettent de produire des flux intenses de neutrons thermiques, qui sont extraits du cœur grâce à des canaux neutroniques, généralement pour des recherches de physique fondamentale. Les qualités modératrices de l'eau lourde permettent d'obtenir des faisceaux de neutrons exempts de neutrons rapides (considérés comme parasites), bien adaptés aux expériences de physique de la matière. À l'inverse, ces réacteurs sont peu adaptés aux tests de matériaux car la présence d'une cuve fermée destinée à contenir l'eau lourde rend difficile l'accès d'équipements au voisinage proche du cœur du réacteur.

- Les réacteurs à eau légère :

Les réacteurs à eau légère, dits de type piscine, comportent les réacteurs à cœur ouvert et les réacteurs où le cœur est enfermé dans un caisson, placés dans les deux cas dans une piscine (tank in pool reactor type). Ces réacteurs, sont des installations polyvalentes généralement utilisées pour l'irradiation de divers matériaux. Il convient de noter que, parmi les réacteurs de recherche exploités actuellement dans le monde, ce sont les plus nombreux. La figure 2.1 ci-après montre le réacteur HFR ${ }^{10}$ situé à Petten aux Pays-Bas, à caisson fermé.
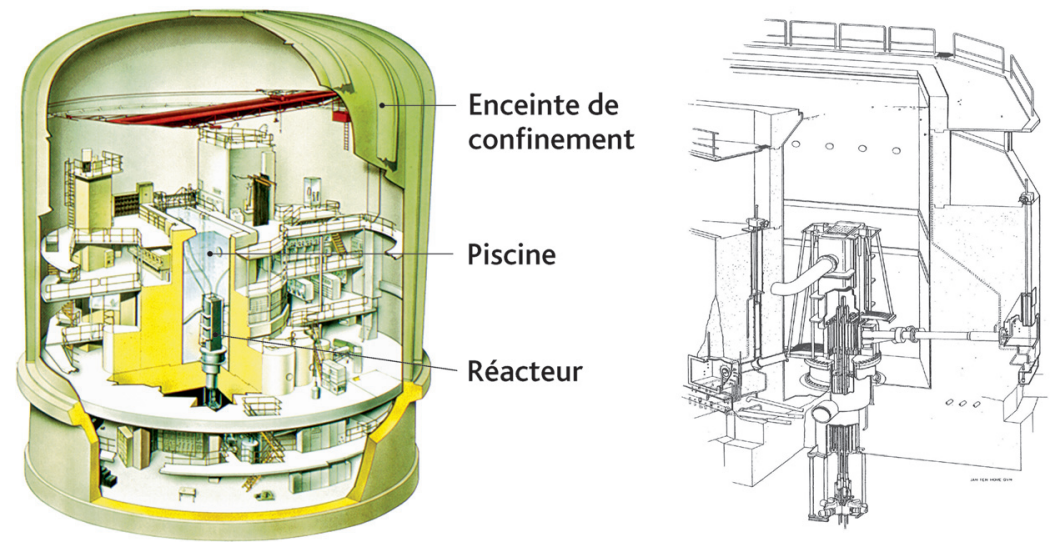

Figure 2.1. Le réacteur HFR de Petten, de type piscine à caisson fermé. @ NRG.

Les réacteurs de type piscine à cœur ouvert permettent en général un accès facile aux emplacements d'irradiation, mais la faible pression d'utilisation (proche de la pression hydrostatique) se traduit par des puissances évacuables moins élevées et donc par des flux neutroniques envisageables plus limités.

Les réacteurs de type piscine comportant un caisson fermé permettent d'atteindre des niveaux de puissance plus élevés que les précédents (avec des pressions plus importantes

10. High Flux Reactor. 
que dans les réacteurs à cœur ouvert), mais leur utilisation pour des irradiations expérimentales est moins facile du fait de la nécessité de dispositifs de traversée du caisson pour accéder au plus près du cœur, les flux neutroniques hors du caisson étant moins importants à cause de l'absorption des neutrons dans le matériau du caisson.

Les réacteurs à eau légère de type piscine à cœur ouvert fonctionnent à de faibles pressions (quelques bars), déterminées par la pression hydrostatique de la hauteur d'eau située au-dessus du cœur (une dizaine de mètres) à laquelle s'ajoute la pression de refoulement des pompes de circulation de l'eau dans le cœur. Pour les réacteurs à eau légère dont le cœur est situé dans un caisson fermé ou les réacteurs à eau lourde, les pressions de fonctionnement peuvent être significativement plus importantes (environ 10 à 20 bars).

Parmi les réacteurs à caisson fermé, la conception adoptée pour le réacteur BR2 ${ }^{11}$, développé par SCK CEN au centre de recherche de Mol en Belgique ${ }^{12}$, en vue de faciliter les expérimentations mérite d'être mentionnée. Ce réacteur, d'une puissance de $100 \mathrm{MW}$, est modéré et refroidi à l'eau légère sous une pression de 22 bars ; le cœur est constitué dans un massif en béryllium. Pour faciliter l'accès aux emplacements d'irradiation, le caisson se présente sous la forme d'un hyperboloïde de révolution (diabolo - figure 2.2) dont le cœur occupe la partie rétrécie. Son couvercle supérieur d'un diamètre environ deux fois plus grand laisse ainsi plus d'espace pour implanter les manchettes de pénétration des dispositifs d'irradiation qui sont légèrement inclinés par rapport à la verticale ${ }^{13}$.

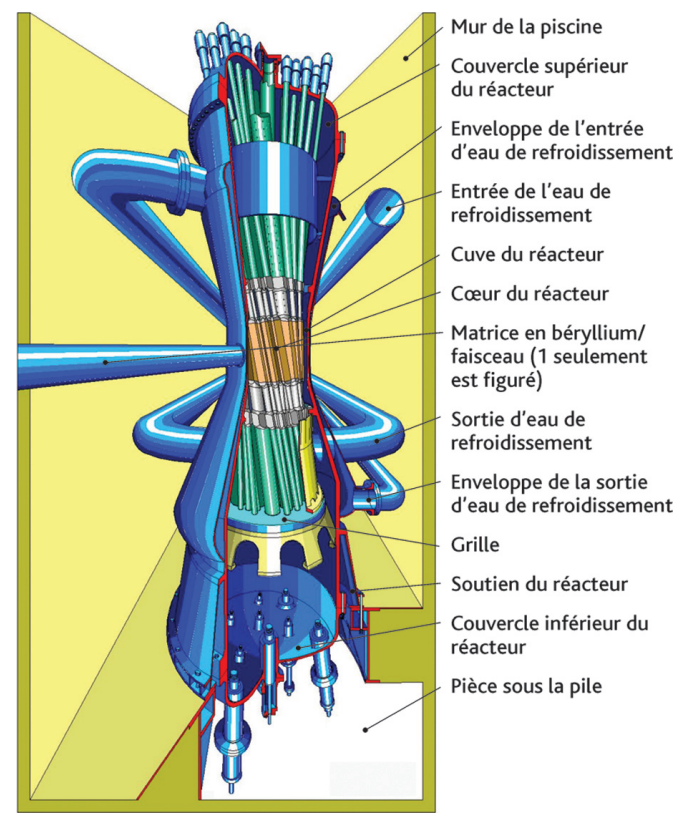

Figure 2.2. Schéma du réacteur BR2. @ SCK CEN.

11. Belgian Reactor 2 .

12. L'un des plus importants producteurs de radioisotopes à usage médical.

13. « Les réacteurs de recherche », Francis Merchie, Encyclopédie de l'énergie, 2015. 


\section{Combustible et cœur des réacteurs de recherche}

De façon générale, les cœurs des réacteurs de recherche sont constitués d'éléments combustibles, d'éléments de contrôle et de sécurité contenant des matériaux absorbant les neutrons, d'éléments réflecteurs permettant de réduire les fuites de neutrons produits dans le cœur du réacteur, et d'emplacements libres pour des irradiations de matériaux.

La description qui suit fait préférentiellement référence aux réacteurs de type piscine.

Pour ces réacteurs, les constituants du cœur sont positionnés sur une grille supportée par une structure métallique placée au fond de la piscine remplie d'eau déminéralisée.

Les éléments combustibles ${ }^{14}$ peuvent se présenter sous la forme d'un assemblage de crayons à base d'oxyde d'uranium, mais plus généralement de plaques dont le combustible est à base d'alliage d'uranium $\left(\mathrm{UAl}_{x}^{15}\right.$ ou $\left.\mathrm{U}_{3} \mathrm{Si}_{2}\right)$ et gainé en alliage d'aluminium (par « colaminage ») (figure 2.3), positionnés (les plaques étant serties) dans une boîte verticale (figure 2.4) canalisant l'eau de refroidissement qui joue également le rôle de

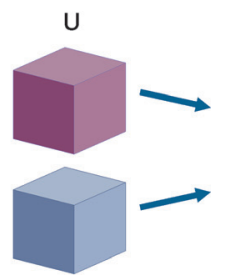

Al, Si ou Mo

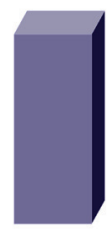

Fusion

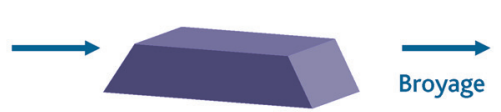

Lingot $\mathrm{Ual}_{x}, \mathrm{U}_{3} \mathrm{Si}_{2}$ ou UMox

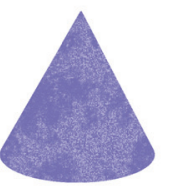

Poudre $\mathrm{UAl}_{x}, \mathrm{U}_{3} \mathrm{Si}_{2}$ ou Umox

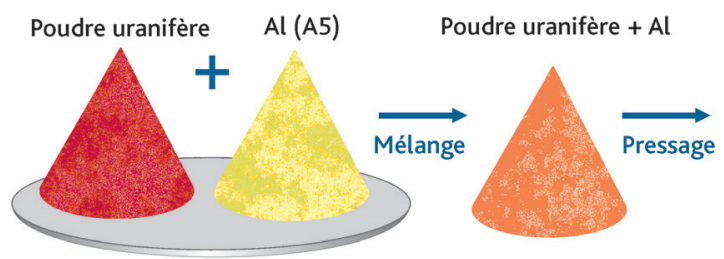

Âme crue

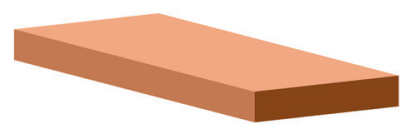

Plaque laminée

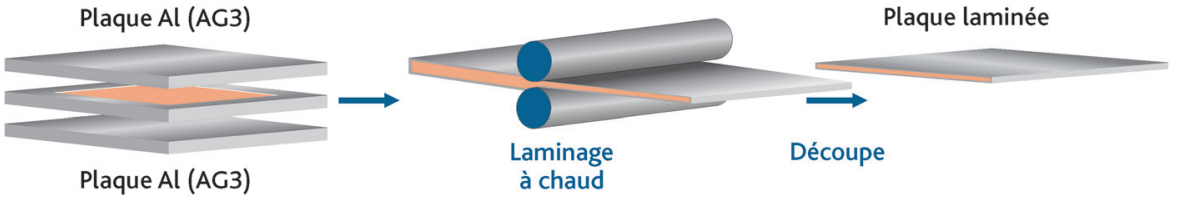

Figure 2.3. Étapes du procédé de fabrication de plaques combustibles par « colaminage ». L'âme combustible est un mélange de poudre combustible $\left(\mathrm{UAl}_{x}, \mathrm{U}_{3} \mathrm{Si}_{2}, \mathrm{UMo}_{\mathrm{x}}\right)$ et d'aluminium issue d'une fusion $\mathrm{U}$ et $\mathrm{Al}$, Si ou Mo. (C) Georges Goué/IRSN.

14. Pour plus de précisions, le lecteur pourra se reporter à l'ouvrage du CEA « Les combustibles nucléaires », monographie de la Direction de l'énergie nucléaire, 2008, notamment au chapitre « Combustibles pour réacteurs de recherche ».

15. La désignation plus couramment utilisée est UAl. Il en est de même pour l'alliage $U_{M} o_{x}$ qui sera évoqué plus loin, dont la désignation courante est UMo. 


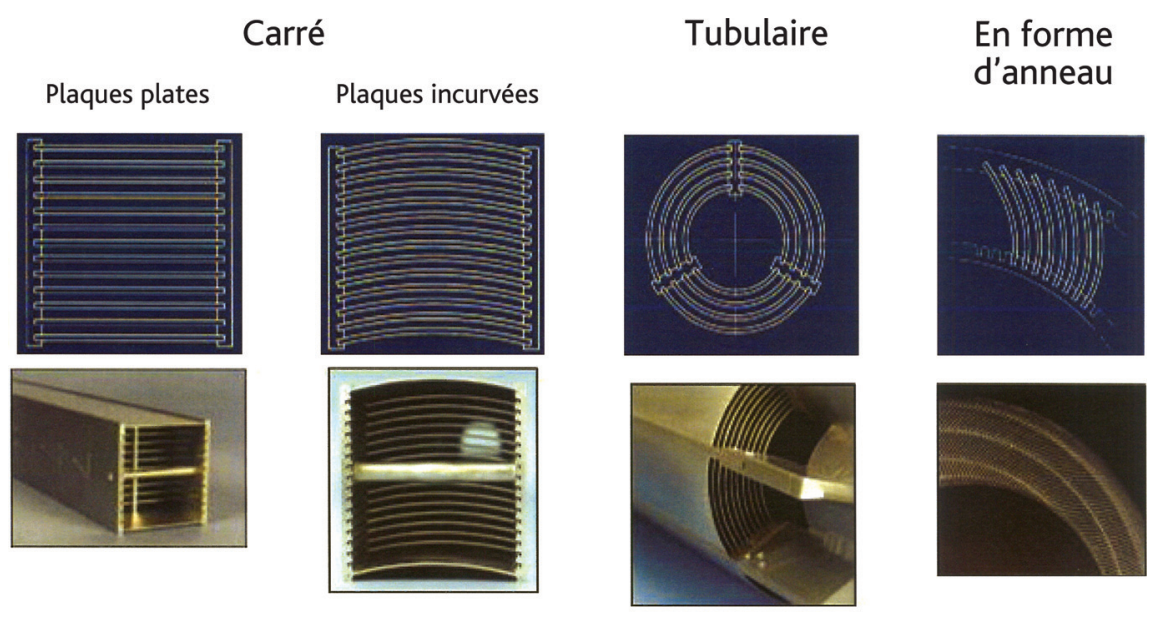

Figure 2.4. Différents types d'éléments ou d'assemblages combustibles de réacteurs de recherche. (C) Orano-CERCA.

modérateur. Le réflecteur du cœur est généralement constitué de béryllium ou de graphite. Il peut aussi être constitué par une cuve à eau lourde entourant le cœur du réacteur. Pour les réacteurs dits « à faisceaux sortis de neutrons », des ouvertures sont aménagées dans les parois latérales de la piscine pour le passage des canaux neutroniques.

Si certains des premiers réacteurs de recherche ont pu fonctionner avec de l'uranium naturel (comportant $0,7 \%$ de son isotope fissile 235), profitant des excellentes propriétés neutroniques de l'eau lourde ou du graphite comme modérateur et réflecteur $^{16}$, la plupart utilisent de l'uranium dont l'enrichissement en isotope 235 varie environ de $20 \%$ jusqu'à $93 \%$.

Parmi les réacteurs de type piscine largement utilisés dans le monde, on peut mentionner ici les réacteurs TRIGA (abréviation de TRaining, Isotope, General Atomics) conçus et construits par la société américaine General Atomics ${ }^{17}$ (voir la figure 2.5 montrant deux de ces réacteurs), dont certaines de leurs caractéristiques, concernant tout particulièrement le combustible et le cœur, méritent d'être mentionnées.

Près d'une quarantaine de réacteurs TRIGA sont actuellement en service. Le plus puissant de ces réacteurs est le réacteur TRIGA du centre nucléaire de Pitesti en Roumanie, dont la puissance thermique est de $14 \mathrm{MW}$. Les autres réacteurs TRIGA ont des puissances thermiques allant d'une centaine de kW jusqu'à $3 \mathrm{MW}$.

Le cœur d'un réacteur TRIGA est positionné au fond d'une piscine contenant de l'eau déminéralisée. Il comporte un réflecteur en graphite ou en béryllium et comprend

16. Réacteurs NRX (National Research eXperimental) et NRU (National Research Universal) au Canada, par exemple.

17. En 1996, General Atomics s'est associé à la société française CERCA (Compagnie pour l'étude et la réalisation de combustibles atomiques, filiale d'Orano) pour créer TRIGA International. CERCA fabrique depuis cette date les assemblages des réacteurs TRIGA. 
généralement une centaine de crayons de combustible contenant des pastilles à base d'un mélange d'uranium et d'hydrure de zirconium ( $\mathrm{UZrH}$ ) gainées d'acier inoxydable ou d'alliage $800^{18}$. Le mélange homogène d'uranium (enrichi à $19,75 \%$ en uranium 235 ) et d'hydrure de zirconium (utilisé comme modérateur) permet d'obtenir un effet important et surtout immédiat de contre-réaction neutronique en cas d'augmentation de la température de ce mélange (coefficient de réactivité d'environ $-10 \mathrm{pcm}^{19} /{ }^{\circ} \mathrm{C}$ ) ${ }^{20}$. Du fait de la bonne stabilité métallurgique de ce combustible et de sa capacité à fonctionner à des températures élevées (habituellement à $750{ }^{\circ} \mathrm{C}$ avec une limite de stabilité du mélange combustible de $1150{ }^{\circ} \mathrm{C}$ ), ainsi que de l'important coefficient de réactivité négatif, le réacteur TRIGA peut être " pulsé » par des injections de réactivité à des niveaux de puissance très élevés (pouvant atteindre, pour les réacteurs TRIGA actuellement en exploitation, $22000 \mathrm{MW}$ ) pendant des fractions de seconde, car l'augmentation rapide de la puissance est rapidement arrêtée par l'effet de réactivité négatif du modérateur. Il est également à noter que le combustible UZrH a un fort potentiel de rétention des produits de fission comparativement aux plaques combustibles à base d'aluminium.
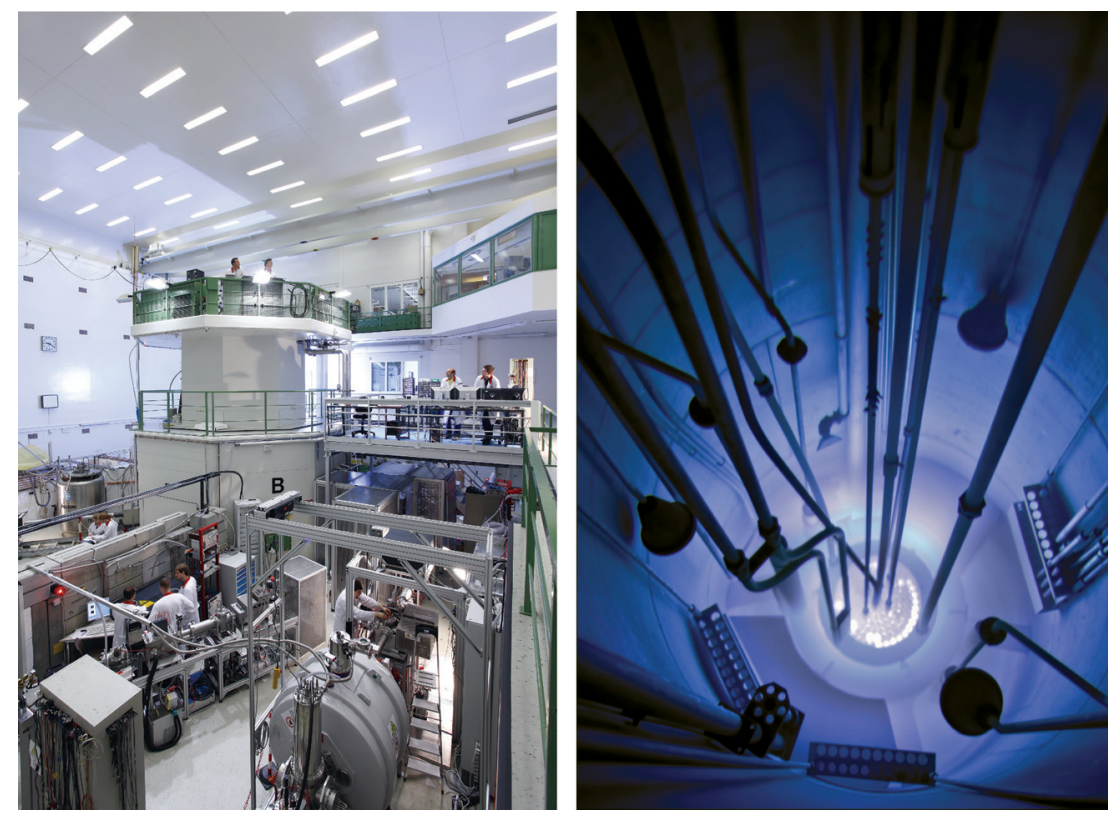

Figure 2.5. À gauche, le réacteur TRIGA de Mainz, Allemagne. @ Thomas Hartmann, Johannes Gutenberg University Mainz ; à droite, le réacteur TRIGA de l'université de l'Oregon, États-Unis. (๐) Oregon State Radiation Center and School of Nuclear Science and Engineering.

18. Il s'agit d'alliages de fer, de nickel et de chrome, qui combinent une bonne résistance à la rupture et une excellente résistance à l'oxydation et à la carburation aux températures élevées et dans de nombreux environnements aqueux.

19. $\mathrm{pcm}$ : pour cent mille.

20. Il s'agit du coefficient $\Delta \mathrm{k} / \mathrm{k} /{ }^{\circ} \mathrm{C}$, représentant la variation relative du coefficient de multiplication des neutrons par élévation de la température d'un degré Celsius. 


\subsection{Situation globale dans le monde}

\subsubsection{Données statistiques}

D'après la base de données RRDB ${ }^{21}$ - données de mai 2018 - de l'Agence internationale à l'énergie atomique (AIEA), 807 réacteurs de recherche ont été construits dans le monde et 23 sont en projet ou en cours de construction. Parmi les réacteurs construits, 430 réacteurs ont été déclassés (plus de la moitié de ces réacteurs sont aux États-Unis), 223 réacteurs sont en service, les 154 autres étant en arrêt de longue durée sans utilisation (ou non encore déclassés). Environ :

- $27 \%$ des réacteurs de recherche ont une puissance (thermique) inférieure à $1 \mathrm{~kW}$;

- $35 \%$ une puissance (thermique) comprise entre $1 \mathrm{~kW}$ et $1 \mathrm{MW}$;

- $38 \%$ une puissance (thermique) supérieure à $1 \mathrm{MW}$.

Parmi les réacteurs en service dans le monde :

- plus de $50 \%$ d'entre eux correspondent à des réacteurs d'irradiation technologique (MTR) et polyvalents (produisant, en outre, des radioisotopes, disposant de « faisceaux sortis de neutrons »...) ;

- environ $20 \%$ d'entre eux sont du type maquette critique de très faible puissance ;

- un peu plus de $10 \%$ d'entre eux sont des petits réacteurs essentiellement dévolus à la formation et à l'entraînement.

La Fédération de Russie possède le plus grand nombre de réacteurs (en service ou en arrêt temporaire) de recherche (54), suivie par les États-Unis (50), la Chine (16), le Japon (9), l'Allemagne (7) et la France (5) ${ }^{22}$. Beaucoup de pays en voie de développement possèdent également des réacteurs de recherche ou en envisagent l'acquisition. Neuf réacteurs de recherche sont en construction dans le monde et quatorze sont en projet.

Malgré l'intérêt croissant porté aux réacteurs de recherche par les pays en voie de développement, le nombre total de ces réacteurs sur le plan mondial diminue d'une façon régulière (cette baisse depuis 2005 correspond en moyenne à l'arrêt d'un réacteur de recherche par an). Ce fait peut être attribué à l'ancienneté de certaines installations qui nécessiteraient souvent d'importants travaux de rénovation ou de modification pour porter leur sûreté à un niveau approprié au vu des pratiques actuelles. Cela peut aussi être dû à l'insuffisance des moyens budgétaires pour en assurer le fonctionnement et la maintenance voire à l'absence de programme d'utilisation. À cet égard, il convient de noter que près de $40 \%$ des réacteurs de recherche sont sous-utilisés (154 réacteurs sont en arrêt de longue durée ou en arrêt permanent).

21. Research Reactors Data Base. Le recensement donné dans cette base tient compte des réacteurs de recherche ne relevant pas que des installations nucléaires civiles.

22. Sont ici comptés dans la base RRDB les réacteurs ISIS, RHF, CABRI, ORPHÉE et MASURCA. 


\subsubsection{Aspects génériques en matière de sûreté nucléaire et de non-prolifération}

Malgré leur diversité de conception et de qualité d'exploitation, des questions importantes de sûreté revêtant un caractère générique ont été soulevées pour de nombreux réacteurs de recherche exploités dans le monde ; ces questions visent essentiellement :

- le maintien du niveau de sûreté du fait du vieillissement des installations (au sens large, y compris l'obsolescence de matériels),

- le management de leur sûreté par les exploitants,

- l'efficacité du contrôle réglementaire de leur sûreté.

Ces éléments ont en particulier été mis en évidence par les différents bilans de sûreté dressés par l'AIEA à l'occasion de diverses réunions et ateliers techniques, sur la base du retour d'expérience de ses activités concernant la sûreté des réacteurs de recherche, incluant les résultats de nombreuses missions de " revue de sûreté ${ }^{23}$ ".

Un autre sujet marquant à caractère générique est la « conversion » des réacteurs de recherche utilisant initialement du combustible très enrichi en uranium 235 afin de lui substituer du combustible moins enrichi (n'excédant pas $20 \%$ d'uranium 235), dans un souci de non-prolifération nucléaire.

La maîtrise du vieillissement et la « conversion » des réacteurs de recherche utilisant du combustible très enrichi en isotope 235 de l'uranium font l'objet de développements ci-après.

En outre, il a été indiqué précédemment que près de $20 \%$ des réacteurs de recherche sont dans une situation d'arrêt prolongé, sans utilisation et sans une claire définition de leur avenir. Cela constitue un sujet de préoccupation de l'AIEA, qui entreprend différentes actions visant, notamment, à optimiser l'utilisation des réacteurs de recherche.

\subsubsection{Maîtrise du vieillissement des réacteurs de recherche}

Il convient de distinguer deux aspects :

- le vieillissement proprement dit qui résulte de divers mécanismes d'endommagement - ou pathologies - susceptibles d'affecter des composants (structures métalliques, ouvrages de génie civil, câbles et autres équipements électriques, etc.) au cours du temps, qui, en dépit des précautions ${ }^{24}$ prises en termes de conception, de construction et d'exploitation, peuvent mener à des dégradations rédhibitoires (fissuration, fragilisation...) ;

23. Il s'agit des Integrated Safety Assessment of Research Reactors (INSARR) (évaluation intégrée de la sûreté des réacteurs de recherche).

24. Notamment sous la forme de marges, correspondant à des « provisions », pour les mécanismes d'endommagement qui peuvent être anticipés et quantifiés. 
- l'obsolescence de matériels au regard de l'état le plus récent des technologies, des normes et des exigences de sûreté.

Les bilans de l'AIEA sur le retour d'expérience font ressortir que le vieillissement et l'obsolescence figurent parmi les causes premières d'incidents survenant dans les réacteurs de recherche dans le monde.

Le vieillissement et l'obsolescence concernent tout particulièrement les réacteurs d'irradiation, dont certains sont utilisés également pour la production de radioisotopes à usage médical.

La décision prise dans un pays de rénover un réacteur de recherche ou de l'arrêter, avec ou sans remplacement par un nouveau réacteur, va dépendre de plusieurs facteurs, en particulier :

- le taux d'utilisation de l'installation ;

- les besoins du pays (ou de la région) en radioisotopes pour des applications médicales ;

- les études expérimentales à réaliser en accompagnement d'un programme électronucléaire national en cours ou envisagé ;

- l'évolution de l'environnement du réacteur (urbain, industriel...) ;

- l'ampleur et le degré de faisabilité des travaux de rénovation nécessaires ainsi que leur coût, y compris ceux pour atteindre un niveau de sûreté satisfaisant.

Il convient de noter que, dans le cas des réacteurs de recherche, il est généralement possible de remplacer l'ensemble de leurs composants, à l'exception des ouvrages de génie civil. Cela a été confirmé par les importantes rénovations effectuées pour différents réacteurs de recherche.

Comme pour les autres types d'installations nucléaires, le vieillissement et l'obsolescence pour les réacteurs de recherche peuvent conduire à une réduction des marges de sûreté (par rapport à des phénomènes redoutés) si de telles évolutions ne sont pas détectées et corrigées à temps.

L'obsolescence peut notamment se traduire par des difficultés à approvisionner des pièces de rechange pour le remplacement de composants importants pour la sûreté.

Le vieillissement peut affecter des équipements importants au point de vue de la sûreté : par exemple la corrosion du cuvelage de la piscine du réacteur, ou encore des tuyauteries de circuits de refroidissement. Il peut aussi accroître le risque de défaillances dites de mode commun ${ }^{25}$ de composants redondants.

$\mathrm{Si}$, pour de nombreux réacteurs de recherche, la plupart des structures, systèmes et composants $\left(\mathrm{SSC}^{26}\right)$ sont remplaçables, une approche systématique de maîtrise du

25. Défaillances de plusieurs composants du fait d'une même cause.

26. Structures, Systems and Components, expression anglaise utilisée notamment dans les normes de l'AIEA. 
vieillissement, associée à un programme effectif permettant d'assurer cette maîtrise, est nécessaire. Cela implique par exemple l'utilisation de matériaux ayant une grande résistance à la corrosion ou de matériaux compatibles entre eux (pour effectuer des soudures, pour des protections radiologiques en piscines ${ }^{27}$, etc.) ou encore l'établissement de programmes de contrôle et de maintenance des équipements, incluant la surveillance d'échantillons représentatifs dédiés au suivi ou à l'anticipation du vieillissement d'équipements importants pour la sûreté. D'une façon générale, tous les paramètres pouvant influencer le vieillissement des installations et entraîner la dégradation de structures, systèmes et composants importants pour la sûreté doivent faire l'objet d'un suivi approprié durant la vie du réacteur.

Il convient que la maîtrise du vieillissement soit mise en place et réalisée de façon proactive et anticipative durant les différentes phases de la vie d'un réacteur de recherche. Par exemple, les modifications apportées à un réacteur de recherche ou à ses dispositifs expérimentaux ne doivent pas venir entraver les inspections et les tests destinés à détecter les signes de vieillissement de structures, systèmes et composants importants pour la sûreté - si possible plutôt à les faciliter. Cet objectif peut être satisfait en rendant (lors de la conception initiale de l'installation) et en conservant (en exploitation) ces matériels accessibles, sans préjudice de l'exposition aux rayonnements ionisants des personnes effectuant les inspections, notamment.

Enfin, le retour d'expérience existant sur le vieillissement, qu'il soit spécifique du réacteur concerné ou générique, incluant aussi le retour d'expérience d'installations industrielles, doit être pris en compte pour la maîtrise de son vieillissement. Il existe sur ce sujet une base de données de l'AIEA, dont l'objectif est d'assurer à l'échelle mondiale un partage des connaissances.

\subsubsection{2 « Conversion » des réacteurs de recherche utilisant du combustible très enrichi en uranium 235}

L'utilisation dans les réacteurs de recherche de combustibles à base d'uranium très enrichi en isotope 235 présente un risque de détournement de cette matière fissile pour un usage non pacifique. Ce risque est d'autant plus important que la faible radioactivité de ces combustibles facilite leur manipulation.

L'uranium très enrichi en isotope 235 diffère de l'uranium naturel ou à faible enrichissement utilisé dans les réacteurs de puissance par sa teneur élevée en cet isotope (elle peut atteindre $93 \%$ ). Il est à cet égard considéré que la teneur maximale « non proliférante » est de $20 \%$, compte tenu des risques associés de détournement ou de vol de combustibles non irradiés et des risques liés à la production de plutonium au cours de l'irradiation, en réacteur, de combustibles faiblement enrichis en isotope 235 .

Dans les années 1950 et 1960, les États-Unis et l'Union soviétique ont commencé à exporter de l'uranium très enrichi en isotope 235 dans le cadre de leurs programmes de

27. Par exemple, le plomb peut provoquer une corrosion de structures en aluminium selon la qualité physico-chimique de l'eau. 
coopération nucléaire dans le domaine civil (en particulier le programme américain Atoms for Peace ${ }^{28}$ lancé en 1954).

En 1978, le Department of Energy ${ }^{29}$ (DOE) des États-Unis a lancé le programme intitulé Reduced Enrichment for Research and Test Reactors ${ }^{30}$ (RERTR) dont le but était de " convertir " les réacteurs de recherche utilisant du combustible très enrichi en uranium 235, et d'origine américaine, à l'utilisation de combustible à faible enrichissement (inférieur à $20 \%$ ). Au milieu des années 1980, ce programme a été étendu pour y inclure les installations de production de radioisotopes, avec en particulier le développement de technologies de production de molybdène 99 pour la médecine nucléaire à l'aide de cibles d'uranium à faible enrichissement en isotope 235 .

Au début des années 1990, le programme a également été étendu, en collaboration avec des instituts russes, aux réacteurs utilisant du combustible très enrichi d'origine russe. Cela a concerné du combustible très enrichi, neuf ou usé, de réacteurs de recherche en Pologne, Serbie, Ukraine et Ouzbékistan, dans le cadre de leur « conversion » à du combustible de faible enrichissement en uranium 235.

Après les attaques terroristes du 11 septembre 2001 sur les tours du World Trade Center à New York, les efforts et les ressources dédiés à la réduction de l'enrichissement en uranium 235 des combustibles utilisés dans les réacteurs de recherche ont été fortement augmentés, notamment avec le lancement par l'administration américaine, en 2004, du programme Global Threat Reduction Initiative ${ }^{31}$ qui a regroupé le programme RERTR avec d'autres initiatives américaines. Cela vise notamment à renforcer les conditions d'autorisation des exportations d'uranium très enrichi en isotope 235 pour les réacteurs de recherche et à récupérer les combustibles nucléaires très enrichis exportés, après leur utilisation, en assurant leur sécurité.

Du lancement du programme RERTR à la fin de l'année 2011, environ 75 réacteurs de recherche (parmi 129 réacteurs de recherche sélectionnés dans le cadre de ce programme pour la conversion, incluant les réacteurs universitaires américains) ont été " convertis » à l'utilisation de combustible à faible enrichissement en uranium 235 ou ont été définitivement arrêtés. L'objectif du programme est de terminer vers 2020 la « conversion » des réacteurs restants, sachant que, pour 28 d'entre eux, la « conversion » suppose la « qualification » d'un nouveau combustible $\mathrm{UMo}^{32}$ de haute densité (environ 7 grammes d'uranium par $\mathrm{cm}^{3}$ ).

Il convient de noter que la majorité des « conversions » dans les pays en voie de développement ont été réalisées en coopération avec l'AIEA et que cette " conversion » a été l'occasion, pour certains d'entre eux, d'une rénovation d'équipements importants pour la sûreté.

28. Des atomes pour la paix.

29. Ministère de l'énergie américain.

30. Réduire l'enrichissement pour les réacteurs d'essais et de recherche.

31. Initiative mondiale de réduction des menaces.

32. Combustible à base d'un alliage d'uranium et de molybdène, dans une matrice environnante d'aluminium (voir la figure 2.3). 


\subsection{Utilisations des réacteurs de recherche et principaux risques associés}

Plusieurs réacteurs de recherche, implantés dans des universités ou dans des organismes de recherche, sont utilisés pour la formation d'étudiants, d'ingénieurs et de personnels de l'industrie nucléaire, incluant les personnels d'exploitation de réacteurs de recherche et de réacteurs de puissance, ou encore d'autorités de sûreté nucléaire.

Les réacteurs de recherche constituent également des outils pour la recherche fondamentale et des recherches appliquées, notamment dans les domaines de la physique nucléaire et des sciences de la matière, ainsi que de l'analyse par activation, de la radiochimie et de la médecine nucléaire. Ils permettent de produire une grande variété de radioisotopes pour des applications médicales ou industrielles, ainsi que pour l'agriculture et la recherche, et de créer des matériaux modifiés par transmutation neutronique ${ }^{33}$ pour l'industrie électronique. Ils permettent aussi de tester divers types de combustibles nucléaires et d'étudier le comportement de différents matériaux sous irradiation ou dans des conditions accidentelles simulées.

Ces diverses utilisations des réacteurs de recherche sont développées dans les paragraphes qui suivent. Le lecteur pourra aussi consulter à ce sujet le rapport établi par l'AlEA en 2007 dressant un panorama détaillé de diverses utilisations des réacteurs de recherche dans le monde ${ }^{34}$.

\subsubsection{Formation}

En principe, tous les réacteurs de recherche peuvent être utilisés pour l'éducation et la formation professionnelle dans le domaine nucléaire. Mais pour des raisons de sûreté et d'accessibilité, les réacteurs de recherche de faible puissance (jusqu'à quelques centaines de kilowatts) sont mieux adaptés aux activités de formation, qui peuvent inclure notamment la réalisation de mesures neutroniques et de mesures de radioprotection, ainsi que la caractérisation d'un cœur de réacteur en établissant la courbe d'efficacité des éléments absorbants et en mesurant le coefficient de température et la distribution de puissance. Ce type de réacteur permet également aux personnes en formation d'acquérir des connaissances et une expérience pratique du pilotage d'un réacteur nucléaire (approche sous-critique, divergence...). Il est évidemment important d'utiliser des configurations de cœur spécifiques présentant une faible réactivité potentielle, permettant de prévenir les accidents de réactivité en cas de fausse manœuvre. Il est à noter également que le réglage des seuils de sécurité, opération répétée fréquemment pour un réacteur dédié à l'enseignement, constitue une opération dont la maîtrise nécessite une attention particulière au point de vue des facteurs humains et organisationnels, notamment de la part des formateurs ou du personnel d'exploitation.

33. Dopage du silicium pour la fabrication de composants électroniques.

34. Technical Reports Series No. 455 : Utilization related design features of research reactors : a compendium, 2007. Le lecteur pourra également consulter l'ouvrage du CEA « Les réacteurs nucléaires expérimentaux », monographie de la Direction de l'énergie nucléaire, 2012. 


\subsubsection{Recherche fondamentale}

Les « faisceaux sortis de neutrons » de réacteurs de recherche permettent d'effectuer des recherches en physique nucléaire ou en physique de la matière condensée et d'étudier des structures cristallines par diffraction des neutrons thermiques. En effet, les neutrons, dont la masse unitaire est voisine de celle d'un atome d'hydrogène et dont la charge électrique est neutre, pénètrent aisément dans la plupart des matériaux et constituent ainsi un outil privilégié d'exploration de la matière. Avec une vitesse de l'ordre de $2200 \mathrm{~m} / \mathrm{s}$, les neutrons thermiques ont une longueur d'onde associée de 0,18 nm (nanomètre), parfaitement adaptée à l'étude des structures cristallines par diffraction puisqu'elle est du même ordre de grandeur que les distances réticulaires dans les cristaux ${ }^{35}$.

Les réacteurs les plus adaptés pour les expériences de diffusion et de diffraction neutroniques et les études de physique des solides ont généralement des puissances thermiques supérieures à une dizaine de mégawatts et des flux neutroniques supérieurs à $10^{14}$ neutrons $/ \mathrm{cm}^{2}$.s.

L'utilisation d'une « source froide » contenant de l'hydrogène ou du deutérium liquide (à une température d'environ $20 \mathrm{~K}$ ) ou d'une « source chaude » contenant du graphite (à une température d'environ $1500 \mathrm{~K}$ ) permet de déplacer le spectre d'énergies des neutrons et d'obtenir pour certaines recherches des longueurs d'ondes plus élevées ou plus faibles. Par ailleurs, l'utilisation de canaux neutroniques, dont la longueur peut atteindre une centaine de mètres, permet d'augmenter le nombre d'expériences installées autour du réacteur.

Les principaux risques associés aux expériences menées auprès des canaux neutroniques sont des risques industriels classiques et des risques d'irradiation pour les expérimentateurs. Des irradiations élevées d'expérimentateurs se sont produites dans différentes installations, soit à la suite de dysfonctionnements d'organes de sûreté (tels que des obturateurs de faisceaux neutroniques, des balises ou des dispositifs de signalisation et de mesures de rayonnements), soit du fait d'un nonrespect de consignes de sécurité par les expérimentateurs. Beaucoup des expérimentateurs concernés provenaient d'organismes extérieurs et ne connaissaient pas nécessairement les différents risques associés aux zones expérimentales dans lesquelles ils travaillaient. À la suite de ces incidents d'irradiation, les exploitants ont pris des dispositions pour mieux sensibiliser les expérimentateurs aux risques associés à leurs zones de travail (par exemple, mise en place à l'entrée de chaque zone expérimentale d'une affiche signalant les risques classiques et radiologiques associés). Des modifications matérielles ont été également réalisées, par exemple pour déclencher une alarme sonore et visuelle en cas d'accès non autorisé dans une zone expérimentale où le débit de dose dépasse une valeur prédéfinie.

Enfin, les risques associés aux "sources froides " et aux "sources chaudes ", notamment ceux d'explosion d'hydrogène ou de deutérium ou d'explosion de vapeur (par interaction entre du graphite et de l'eau), qui sont susceptibles d'affecter le cœur d'un

35. « Les réacteurs de recherche », Francis Merchie, Encyclopédie de l'énergie, 2015. 
réacteur ou ses «barrières » de confinement, sont à traiter dans le cadre de la démonstration de sûreté ${ }^{36}$ de ce réacteur.

\subsubsection{Irradiations expérimentales}

Les réacteurs de recherche ayant une puissance thermique supérieure à une dizaine de mégawatts constituent un outil de choix pour l'étude et la qualification de combustibles nucléaires, de matériaux de structures et de composants des réacteurs de puissance (cuves, équipements internes, absorbants neutroniques, etc.). Les irradiations peuvent être réalisées dans des conditions neutroniques et thermohydrauliques représentatives du fonctionnement normal des réacteurs de puissance ou, pour certains réacteurs d'irradiation le permettant, dans des conditions représentatives de situations accidentelles de réacteurs de puissance correspondant à des insertions de réactivité ou à des réductions du débit de refroidissement du cœur du réacteur, sans aller jusqu'à des conditions sévères (fusion de combustible) qui sont spécifiquement étudiées avec les réacteurs d'essais de sûreté.

L'utilisation de réacteurs de recherche pour la réalisation de telles irradiations présente des avantages importants par rapport aux réacteurs de puissance :

- leur flux neutronique plus élevé permet d'obtenir les doses d'irradiation prévues dans des délais plus courts (par exemple pour l'étude du vieillissement de matériaux sous irradiation) ;

- la possibilité de mieux instrumenter les échantillons à étudier permet de mesurer par exemple, de façon plus précise, des températures, des pressions et les flux neutroniques ;

- la possibilité de rapprocher des échantillons de combustibles à étudier du cœur du réacteur en utilisant un " dispositif à déplacement » (télescopique) permet de simuler des rampes ${ }^{37}$ lentes de puissance (figure 2.6) ;

- les risques sont plus faibles en cas d'accident lors du déroulement d'une irradiation expérimentale.

Les irradiations sont généralement réalisées dans des dispositifs expérimentaux contenant les échantillons de combustible ou les matériaux à irradier dans des conditions bien définies en termes de température, de pression, de flux neutronique, de fluide environnant (gaz neutre, eau liquide ou sous forme de vapeur, sodium liquide, etc.).

Chaque dispositif d'irradiation doit faire l'objet d'un examen spécifique en termes de sûreté, traitant en particulier des risques associés aux interactions possibles entre le dispositif et le réacteur, à savoir l'impact possible du dispositif sur la sûreté du réacteur et des autres " expériences », ainsi que l'impact, sur la sûreté du dispositif, des

36. Les dispositions qu'un exploitant a retenues en vue d'assurer un niveau de sûreté approprié de son installation doivent être décrites dans des documents qui visent à présenter les justifications du bien-fondé de ces dispositions et de leur caractère suffisant, ce qui est convenu d'appeler " démonstration de sûreté ».

37. Évolutions lentes de puissance au regard de transitoires rapides (" pulses »). 


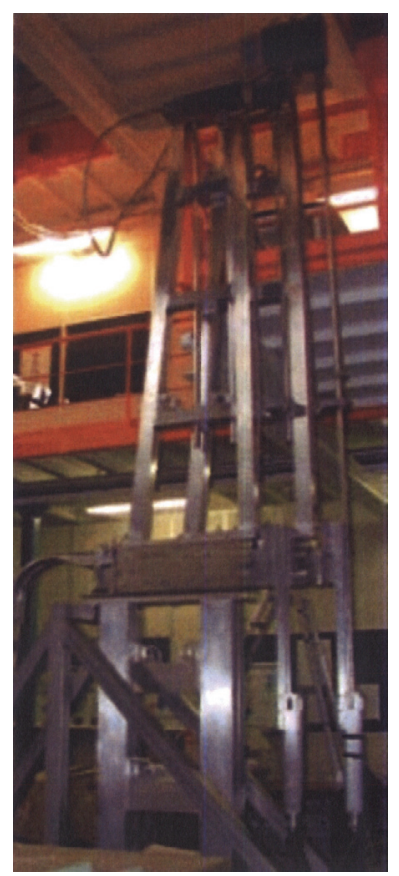

Figure 2.6. Dispositif télescopique du réacteur OSIRIS servant à réaliser des rampes lentes de puissance, d'après le rapport AIEA 455. (C) DR.

événements (liés au réacteur lui-même ou aux événements de type " agressions » internes ou externes) retenus pour la conception du réacteur et la démonstration de sûreté associée.

Parmi les dispositifs expérimentaux utilisés, on peut mentionner ici les capsules d'irradiation - qui ne sont pas instrumentées - et les boucles d'irradiation, à eau, à gaz ou à métal fondu (par exemple du sodium pour la filière des réacteurs à neutrons rapides refroidis au sodium).

Les capsules d'irradiation comportent en général deux « barrières » (enveloppes métalliques) externes entre la matière radioactive et l'extérieur, dont l'étanchéité est surveillée par un suivi de la pression de la lame de gaz (azote ou hélium) présente entre les deux « barrières ». La sûreté des capsules repose sur des choix de conception justifiés par des calculs d'échauffement thermique et de montée en pression de ses différents constituants lors des irradiations ainsi que sur la compatibilité chimique ${ }^{38}$ des matériaux mis en jeu. Des incidents mettant en jeu des capsules d'irradiation se sont produits, par éclatement ou perte d'étanchéité de "barrières ", qui ont conduit à une contamination de la piscine ou des structures du réacteur, voire à l'irradiation d'expérimentateurs.

Les boucles d'irradiation permettent d'étudier le comportement de combustibles nucléaires utilisés dans les différentes filières de réacteurs nucléaires, dans des conditions

38. Risques de formation d'eutectiques, de corrosion galvanique (acier au contact de l'aluminium), etc. 
représentatives des conditions de fonctionnement normales, incidentelles ou accidentelles des réacteurs de puissance. Ces boucles qui, comme les capsules d'irradiation, sont dotées d'enveloppes jouant un rôle de « barrière », s'en distinguent par l'existence d'un circuit de refroidissement des échantillons étudiés. Comme cela a été indiqué plus haut, le fluide de refroidissement peut être de l'eau (sous pression), un gaz ou un métal fondu.

Les différents paramètres d'un dispositif expérimental d'irradiation (pressions, températures, débits de refroidissement dans le cas des boucles, etc.) sont suivis en continu au cours des irradiations. Des actions de sécurité déclenchent l'arrêt automatique du réacteur ou la mise en sécurité du dispositif lui-même (par exemple, en cas de dépressurisation d'une boucle en pression), dès lors que des seuils prédéfinis sont dépassés.

Les principaux risques associés aux boucles d'irradiation incluent :

- le risque de contamination et d'irradiation de personnels en cas de perte d'étanchéité des « barrières » de la boucle ;

- le risque d'endommagement des structures de la boucle et d'émission de projectiles pouvant affecter la sûreté du réacteur en cas de fusion de l'échantillon de combustible testé suivie éventuellement d'une explosion de vapeur (la fusion de l'échantillon peut faire partie des objectifs recherchés pour certaines expériences).

Des dispositions sont prises pour prévenir ces risques et en limiter leurs conséquences.

\section{\#FOCUS}

\section{Quelques réacteurs plus particulièrement dédiés à des essais de sûreté}

- CABRI (centre d'études de Cadarache, France)

Essais relatifs au comportement de combustibles nucléaires en cas d'insertions rapides de réactivité (réacteurs à eau sous pression [REP], réacteurs à neutrons rapides refroidis au sodium $[\mathrm{RNR}]$ ).

- SCARABEE (centre d'études de Cadarache, France) - ce réacteur a été arrêté et démantelé

Essais en support à l'étude d'accidents de fusion par bouchage d'assemblage dans les RNR.

- PHEBUS (centre d'études de Cadarache, France)

Essais relatifs aux accidents de refroidissement des REP et aux transferts de produits de fission associés. 
- NSRR (Nuclear Safety Research Reactor ${ }^{39}$, Tokai Mura, Japon)

Essais en support à l'étude d'insertions rapides de réactivité pour les combustibles de RNR et de réacteurs refroidis à l'eau légère.

- TREAT (Transient Reactor Test Facility ${ }^{40}$ - Idaho National Laboratories, Idaho Falls, États-Unis)

Essais en support à l'étude d'insertions rapides de réactivité pour divers combustibles (RNR, réacteurs refroidis à l'eau légère...).

\subsubsection{Applications médicales}

\subsubsection{Production de radioisotopes}

Les radioisotopes que les réacteurs de recherche permettent de produire sont utilisés dans de nombreux domaines incluant notamment la médecine nucléaire, l'industrie, l'agriculture et la recherche.

L'utilisation de radioisotopes à des fins médicales est globalement en forte croissance. On compte chaque année plus de 30 millions d'examens et de traitements de cancers dans le monde. Le molybdène $99\left({ }^{99} \mathrm{Mo}\right)$ est un exemple de radioisotope très fréquemment utilisé. Il sert à la préparation de générateurs de technétium $99 \mathrm{~m}$, qui est produit par désintégration $\beta$ du molybdène 99 (période de 2,75 jours). Le molybdène 99 est un produit de fission obtenu par l'irradiation de petites plaques de combustible UAI, l'uranium pouvant avoir différents enrichissements en isotope 235. Dans certains réacteurs de recherche, le ${ }^{99}$ Mo est produit par capture neutronique dans des cibles enrichies en ${ }^{98} \mathrm{Mo}$.

Les principaux réacteurs producteurs de ${ }^{99}$ Mo dans le monde sont des réacteurs anciens (BR2 en Belgique, HFR aux Pays-Bas, NRU au Canada, SAFARI ${ }^{41}$ en Afrique du Sud - OSIRIS en France a été arrêté à la fin de 2015) qui nécessitent souvent des arrêts fréquents pour des opérations de maintenance, ce qui peut engendrer des risques de pénurie mondiale de ${ }^{99} \mathrm{Mo}$.

Les risques associés à la production de radioisotopes dans les réacteurs de recherche incluent naturellement les risques de contamination et d'irradiation du personnel d'exploitation ainsi que le risque de rejets radioactifs dans l'environnement.

\subsubsection{Thérapie de tumeurs cancéreuses par capture neutronique}

Plusieurs réacteurs de recherche sont utilisés pour traiter des tumeurs (mélanomes, tumeurs du cerveau). La méthode utilisée, connue sous l'acronyme BNCT (Boron

39. Réacteur de recherche en sûreté nucléaire.

40. Installation d'essai pour l'étude de transitoires en réacteurs.

41. South African Fundamental Atomic Research Installation 1. 
Neutrons Capture Therapy ${ }^{42}$ ), est fondée sur l'absorption de neutrons par le bore 10. Elle consiste à injecter une solution de bore 10 dans la tumeur à traiter et à l'irradier par un faisceau de neutrons provenant du réacteur. L'absorption de neutrons par le bore 10 conduit à l'émission de particules alpha qui sont très ionisantes. Les cellules cancéreuses sont détruites par ces particules dont le parcours est du même ordre de grandeur que le diamètre des cellules.

Des efforts de recherche restent nécessaires pour réduire les durées d'irradiation des patients et diminuer les doses reçues par les cellules saines.

\section{\#FOCUS}

\section{Radionucléides artificiels produits dans des réacteurs de recherche et utilisés dans le secteur médical}

- Techniques de diagnostic :

Technétium 99m (issu de molybdène 99), xénon 133 , tritium $\left({ }^{3} \mathrm{H}\right)$, carbone 14 , ruthénium 97 , iode 125 ;

- Traitements thérapeutiques :

- émetteurs $\beta$ (pour des synovites, resténoses (pathologies artérielles), soins palliatifs (cancers osseux) : yttrium 90, strontium 90, rhénium 186, erbium 169, cuivre 64, samarium 153,

- émetteurs $\gamma$ (pour les cancers) : cobalt 60, iridium 192,

- émetteur $\beta$ et $\gamma$ (pour les cancers de la thyroïde, des hyperthyroïdies): iode 131.

\subsubsection{Analyse par activation}

L'analyse par activation est une méthode permettant de déterminer avec précision des traces d'impuretés dans des échantillons de matériaux pour lesquels une haute pureté chimique est recherchée. Elle est fondée sur la transformation de noyaux atomiques stables en noyaux radioactifs par irradiation neutronique de l'échantillon testé et sur la mesure des rayonnements émis par les radionucléides formés dans le matériau irradié.

L'analyse par activation constitue le domaine d'utilisation le plus fréquent des réacteurs de recherche. Pratiquement n'importe quel réacteur ayant une puissance excédant une vingtaine de kilowatts est capable de fournir des flux de neutrons suffisants pour effectuer de telles analyses. L'utilisation de tubes hydrauliques ou pneumatiques

42. Thérapie de capture de neutrons par le bore. 
reliant le réacteur aux laboratoires d'analyses permet de mesurer des éléments ayant une courte période radioactive.

Du point de vue de la sûreté, les risques associés à cette utilisation des réacteurs de recherche sont généralement faibles. Il s'agit des risques de contamination de locaux ou de personnes dans les laboratoires d'analyses, de risques d'irradiation à la suite du coincement d'une navette contenant une capsule d'irradiation envoyée dans un tube hydraulique ou pneumatique, ainsi que de risques de contamination de structures internes du réacteur en cas d'échauffement excessif d'échantillons irradiés entraînant la perte de leur intégrité et la destruction de capsules d'irradiation. Les dispositions à prendre pour éviter les situations précitées et pour en limiter les conséquences, ainsi que la liste des matériaux dont l'irradiation est interdite dans le réacteur (comme par exemple le mercure à cause de ses propriétés corrosives), doivent être indiquées dans les règles d'exploitation de l'installation.

\subsubsection{Applications industrielles}

Les applications industrielles des réacteurs de recherche sont très nombreuses. Seules trois d'entre elles, très courantes, seront mentionnées ci-après.

Les « faisceaux sortis » de neutrons thermiques permettent d'effectuer des neutronographies de divers objets. La technique de contrôle non destructif par neutronographie, qui est fondée sur la propriété des neutrons d'être arrêtés par des noyaux légers, est complémentaire de celle par radiographie, car elle permet d'examiner de faibles épaisseurs d'éléments légers et de fortes épaisseurs d'éléments lourds. La neutronographie est utilisée pour des contrôles non destructifs dans les domaines de l'aéronautique et de l'espace (par exemple pour le contrôle des dispositifs pyrotechniques équipant les lanceurs de fusée), ainsi que dans le domaine nucléaire. On peut citer à titre d'exemple l'installation de neutronographie des combustibles irradiés qui était associée au réacteur PHENIX à Marcoule, composée principalement d'un petit réacteur constitué d'une cuve contenant une solution fissile (nitrate d'uranyle), équipée d'un circuit de refroidissement, d'un réflecteur fixe et d'un réflecteur mobile permettant, par rapprochement avec la cuve, d'initier la réaction en chaîne.

L'irradiation neutronique de lingots de silicium modifie ce matériau en y générant d'une façon uniforme du phosphore 31, ce qui le rend semi-conducteur. Cette méthode permet d'obtenir une très bonne distribution de la résistivité dans les lingots de silicium utilisés pour la fabrication de composants pour l'industrie électronique tels que les diodes et thyristors.

L'irradiation par des neutrons rapides permet d'obtenir une coloration du topaze utilisé en bijouterie. Cette activité est interdite dans beaucoup de pays, mais est encore effectuée dans certains réacteurs de recherche dans le monde. 